Check for updates

Cite this: RSC Adv., 2018, 8, 26230

\title{
Facile tailoring of the electrical transport in representative hole transport materials by molecular doping $\dagger$
}

\begin{abstract}
Bixin Li, (D) *a Chenyang Zhao, (D) ${ }^{b}$ Shiyang Zhang ${ }^{a}$ and Min Zhen ${ }^{a}$
$N, N^{\prime}$-Diphenyl- $N, N^{\prime}$-bis(1-naphthyl)-1,1'-biphenyl-4,4'-diamine (NPB) and 4,4', $4^{\prime \prime}$ - tris ( $N$-3-methylphenyl$N$-phenylamine) triphenylamine ( $m$-MTDATA) are widely used as hole transport materials in organic optoelectronic devices. In the present article, the hole transport properties of blends of NPB and $\mathrm{m}$ MTDATA compared with the pristine materials are investigated using admittance spectroscopy and considering temperature dependent current-voltage characteristics and electroluminescent characteristics. It has been found that $m$-MTDATA dramatically lowers the carrier mobility in the NPB matrix to a large extent by enhancing the total density of traps and results in more dispersive transport. However, by introducing NPB into $m$-MTDATA the hole mobility is nearly unchanged in comparison with pristine $m$-MTDATA film. These differences are attributed to two different charge transport mechanisms, trapping and scattering. Obtained quantitative information regarding the charge transport parameters could help to extend optimization strategies for the fabrication of new organic optoelectronic devices by enabling the facile tailoring of the charge transport process.
\end{abstract}

Received 30th April 2018

Accepted 9th July 2018

DOI: $10.1039 / \mathrm{c} 8 \mathrm{ra0} 3707 \mathrm{~h}$

rsc.li/rsc-advances level of the matrix material while doping with a dopant with a lower-lying HOMO level generates no additional trap states. ${ }^{12-14}$ These two types of dopant are defined as trap and scatterer, respectively. Subsequently, the influence of charge trapping and scattering on the hole transport properties of $N, N^{\prime}$ di(naphthalene-1-yl)- $N, N^{\prime}$-diphenyl-benzidine (NPB) has been studied by So et al. via the time-of-flight (TOF) technique. ${ }^{15,16}$ The charge transport properties were analyzed using the Gaussian disorder model and they revealed that the traps significantly enhance the energetic disorder. For the scattering, it changes a little. The effects of traps and scatterers with various energy level differences have also been investigated. ${ }^{17}$ In these studies, the mobilities were determined using the TOF technique which generally requires several micron thick films due to the finite penetration depth of the incident light when retaining a well-defined flight distance. ${ }^{18}$ In particular, the TOF transients become featureless and the carrier transit time is difficult to determine in highly dispersive materials. ${ }^{19}$ Alternatively, admittance spectroscopy (AS) is a powerful technique for investigating the hole and electron mobilities in organic materials with the advantages of full automatic measurements and no specific requirements for the sample thickness. ${ }^{\mathbf{5 , 9 , 1 0 , 1 9 - 2 5}}$ It is superior to TOF for investigating the charge transport in dispersive materials. Furthermore, the space-charge-limited current (SCLC) model with exponentially distributed traps is commonly used to describe the current-voltage $(I-V)$ characteristics in organic disordered semiconductors with an estimation of the energy and density of traps. ${ }^{26,27}$ So how the trap densities are influenced upon doping will be revealed. 
In the present work, we investigate the effects of intentional doping on NPB and $4,4^{\prime}, 4^{\prime \prime}$-tris $(N-3$-methylphenyl- $N$-phenylamine) triphenylamine ( $m$-MTDATA) by considering $I-V$ and capacitance-frequency $(C-f)$ characteristics. In NPB-doped $m$ MTDATA and $m$-MTDATA-doped NPB films, the mobilities and dispersion parameters as well as trap densities are determined and compared with those of pristine materials. These obtained physical quantities show that the transport properties can be tailored facilely.

\section{Experimental}

NPB and $m$-MTDATA were chosen for this investigation. The HOMO levels of $m$-MTDATA and NPB are $5.1 \mathrm{eV}$ and $5.4 \mathrm{eV}$, respectively. Therefore, the introduction of hole traps is expected when doping $m$-MTDATA into NPB, while NPB would behave as scattering centers in an $m$-MTDATA matrix. ${ }^{13,15}$ All organic materials were purchased from Nichem and used without further purification.

The devices were fabricated by thermal evaporation of the organic materials on indium tin oxide (ITO)-coated glass substrates. The substrate was thoroughly cleaned in an ultrasonic bath using detergents and then de-ionized water. The surface of the ITO substrate was treated with oxygen plasma for 2 min to enhance its work function. All films were deposited under a high vacuum (pressure $<10^{-4} \mathrm{~Pa}$ ). The doped films were deposited using a co-evaporation process with a doping concentration of $4 \%$ by weight. The thickness was controlled in situ using a quarter crystal monitor and calibrated using a Dektak 6M Profiler (Veeco). The aluminum (Al) electrodes were deposited on the organic films through shadow masks with an active area of $16 \mathrm{~mm}^{2}$.

For the measurements of the temperature dependent $I-V$ characteristics, the device structure is ITO/molybdenum oxide $\left(\mathrm{MoO}_{3}\right)(5 \mathrm{~nm}) /$ active layers $(120 \mathrm{~nm}) / \mathrm{MoO}_{3}(5 \mathrm{~nm}) / \mathrm{Al}$, where $\mathrm{MoO}_{3}$ was used to facilitate hole injection from ITO and suppress electron injection from $\mathrm{Al} .^{5}$ For the AS measurements, the samples had the same structure as mentioned above but the thicknesses were between 120 and $1400 \mathrm{~nm}$ to efficiently derive mobilities in different samples due to the limitation of the maximum frequency in the impedance analyzer.

The $I-V$ characteristics were investigated using a Keithley 2400 sourcemeter integrated with a vacuum cryostat (Optistat DN-V, Oxford Instruments). The admittance was measured at room temperature using an Agilent E4980A precision LCR meter in the frequency range of $20 \mathrm{~Hz}$ to $2 \mathrm{MHz}$ with the oscillation amplitude of the ac voltage kept at $100 \mathrm{mV}$. The luminance-voltage $(L-V)$ characteristics were recorded using a Keithley 2400 sourcemeter and a Keithley 2000 multimeter with a calibrated silicon photodiode.

\section{Results and discussion}

Fig. 1 shows the current density-voltage $(J-V)$ characteristics of the hole-only devices for pure and doped films at room temperature. It can be clearly seen that for a given driving voltage the current density decreases around three or four

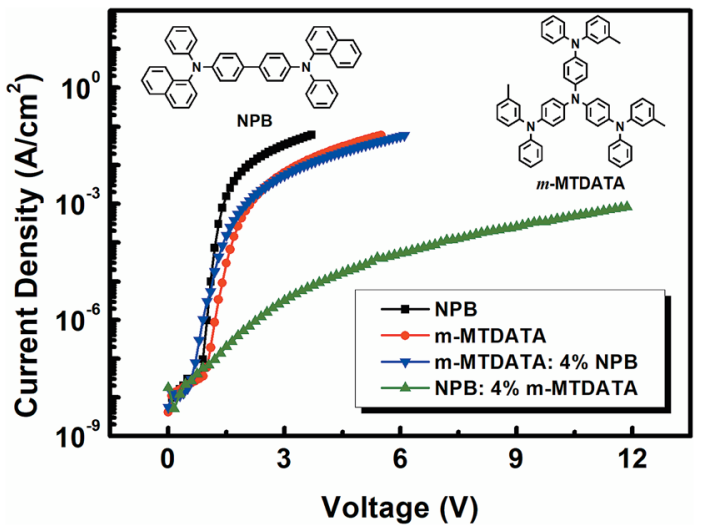

Fig. 1 Current density-voltage characteristics of the hole-only devices. The chemical structures of NPB and $m$-MTDATA are also shown here.

orders of magnitude when $m$-MTDATA is doped into NPB. In particular, the device based on $m$-MTDATA:NPB exhibits slightly reduced, or nearly identical, $J-V$ characteristics compared with those of pristine $m$-MTDATA. For example, at a voltage of $3 \mathrm{~V}$, the current density is $0.034 \mathrm{~A} \mathrm{~cm}^{-2}$ for pure $\mathrm{NPB}$, which reduces sharply to $3.2 \times 10^{-6} \mathrm{~A} \mathrm{~cm}^{-2}$ in m-MTDATA doped NPB film. Meanwhile, the current densities of pristine $m$-MTDATA and NPB doped $m$-MTDATA films are $0.0064 \mathrm{~A} \mathrm{~cm}^{-2}$ and $0.0055 \mathrm{~A}$ $\mathrm{cm}^{-2}$, respectively. The variations in the $J-V$ characteristics implies the different mobilities in doped films, which are related to the charge transport hopping process via the dopant molecules.

AS measurements were done to infer the hole transport properties based on the measurement of the frequencydependent capacitance of an organic film. In this method, the current response, $i_{\text {ac }}$, to a small oscillating voltage, $v_{\text {ac }}$, is monitored. The complex admittance, $Y_{\mathrm{ac}}$, is related to $i_{\mathrm{ac}}$ and $v_{\mathrm{ac}}$ as follows:

$$
Y_{\mathrm{ac}}=\frac{i_{\mathrm{ac}}}{v_{\mathrm{ac}}}=G(\omega)+i B(\omega)=G(\omega)+i \omega C(\omega)
$$

where $G$ is the conductance, $B$ is the susceptance, $C$ is the capacitance and $i^{2}=-1$. In addition, the complex admittance, $Y$, can be deduced analytically and written $\operatorname{as}^{\mathbf{2 0}}$

$$
\begin{aligned}
& Y(\Omega)= \\
& \frac{\varepsilon \varepsilon_{\mathrm{o}} A}{\tau_{\mathrm{dc}} d}\left\{\frac{\Omega^{3}}{2 i[0.75 \tilde{\mu}(\Omega)]^{2}\left[1-\exp \left(-\frac{i 4 \Omega}{3 \tilde{\mu}(\Omega)}\right)\right]+1.5 \tilde{\mu}(\Omega) \Omega-i \Omega^{2}}\right\}
\end{aligned}
$$

where $d$ and $A$ are the thickness and the area of the sample, respectively, $\varepsilon$ is the dielectric constant of the material and $\varepsilon_{\mathrm{o}}$ is the permittivity of free space. In the above equation, $\tau_{\mathrm{dc}}$ is the average hole transit time in the absence of the ac signal, and $\Omega=\omega \tau_{\mathrm{dc}}$. The normalized mobility is defined as $\tilde{\mu}(\Omega)=\mu(\Omega) /$ $\mu_{\mathrm{dc}}$, where $\mu_{\mathrm{dc}}=d^{2} /\left(V \tau_{\mathrm{dc}}\right)$ is the dc hole mobility in the absence of the ac field. To derive the carrier mobility, the imaginary part of $Y$, i.e. the susceptance in the frequency domain, can be 

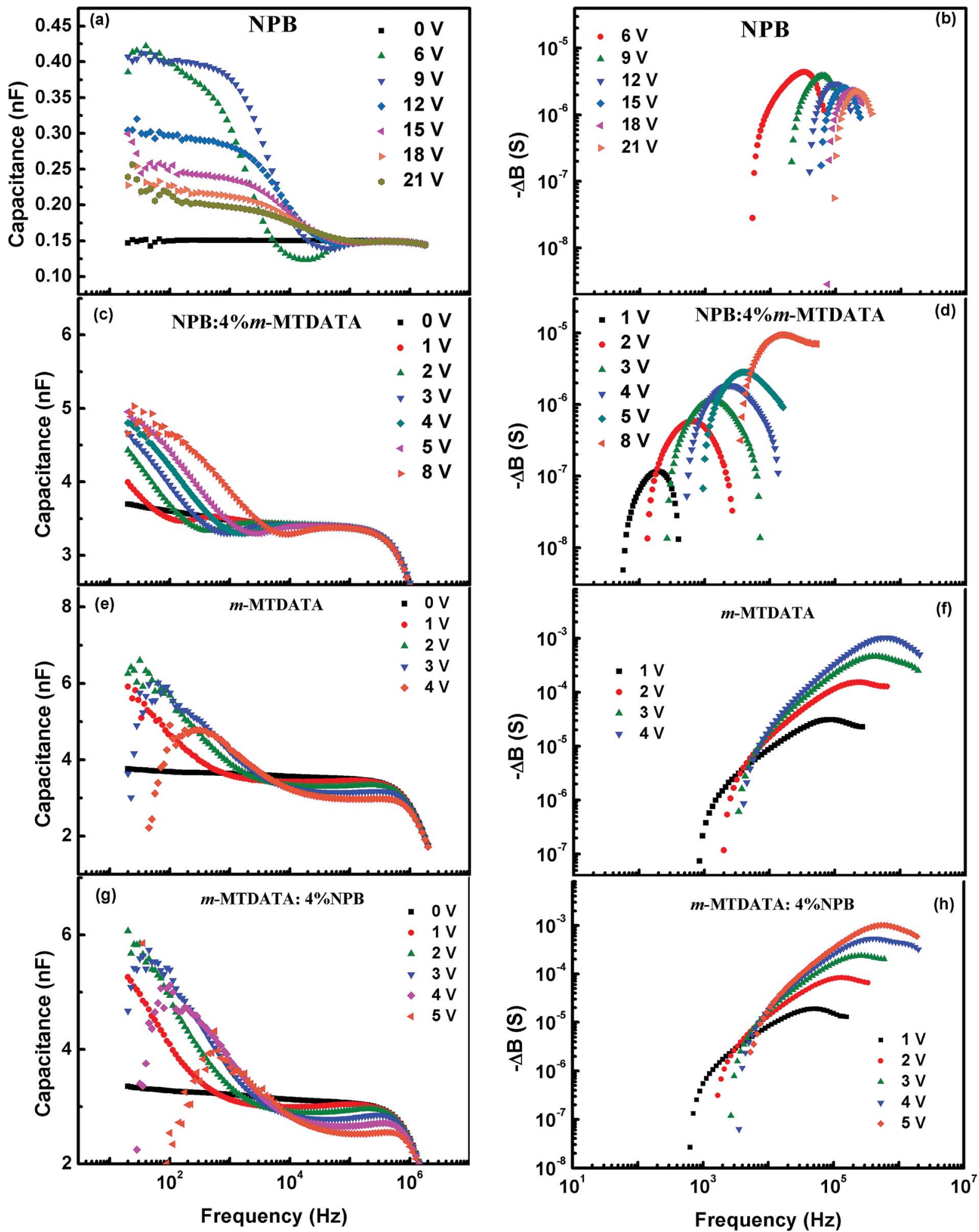

Fig. 2 Frequency dependences of capacitance and $-\Delta B$ for hole-only devices of (a and b) NPB, (c and d) NPB:4\% $m$-MTDATA, (e and f) $m$ MTDATA, ( $g$ and $h$ ) $m$-MTDATA:4\% NPB at room temperature.

analyzed. In particular, the negative differential susceptance of the device can be defined as $-\Delta B=-2 \pi f\left(C-C_{\text {geo }}\right)$. By plotting $-\Delta B$ as a function of frequency, a characteristic frequency $f_{\mathrm{r}}$ at which a maximum of $-\Delta B$ occurs is correlated with the average hole transit time via $f_{\mathrm{r}}=0.56 / \tau_{\mathrm{dc}}{ }^{20}$ Consequently the carrier mobility can be extracted by the following equation:

$$
\mu_{\mathrm{dc}}=\frac{f_{\mathrm{r}} d^{2}}{0.56\left(V-V_{\mathrm{bi}}\right)}
$$


Fig. 2 depicts a series of corresponding $C-f$ and $-\Delta B-f$ plots for four devices. It can be observed that at zero bias voltage, the capacitances are nearly frequency independent and equal to the geometrical capacitance. As bias increases, the capacitance becomes frequency dependent. Due to hole trapping, the excess capacitance can be observed at lower frequencies. With increasing frequencies, a clear valley-like response appears in each $C-f$ curve. Each device shows a minimum capacitance in the intermediate frequency region and then the capacitances tend to their respective geometrical capacitances at high frequency. By replotting the data as $-\Delta B$ versus frequency, the peak $f_{\mathrm{r}}$ can be clearly observed. The shift of $f_{\mathrm{r}}$ to higher frequencies with increasing voltage indicates the field dependence of the mobility.

Fig. 3 shows the mobilities derived from $f_{\mathrm{r}}$ using eqn (3). It can be clearly seen that the mobilities follow the Poole-Frenkel dependence. $^{28}$ The hole mobilities for the pristine NPB and $m$ MTDATA film are in the ranges of (2-4) $\times 10^{-4} \mathrm{~cm}^{2} \mathrm{~V}^{-1} \mathrm{~s}^{-1}$ and $(2-4) \times 10^{-5} \mathrm{~cm}^{2} \mathrm{~V}^{-1} \mathrm{~s}^{-1}$, respectively. In the case of $\mathrm{m}$ MTDATA-doped NPB, the hole mobility reduces dramatically by four orders of magnitude. Meanwhile, for $m$-MTDATA doped with NPB, there is only a slight reduction in mobility or the values are nearly the same. This phenomenon suggests that traps and scatterers have different effects on hole transport mechanisms..$^{15,16}$ In the case of trapping, the mobile holes may be trapped in the generated trap level and then de-trapped by thermal energy. These processes will lead to a reduction in the mobility due to the additional trap residing time. In the case of scattering, the holes may be hampered by the dopant with a lower-lying HOMO level due to collisions. However, holes may transport nearly unrestrainedly in the film.

The immobilization of carriers for time periods by trapping sites that are distributed in energy gap leads to a broad distribution of transit times, rendering the transport more dispersive. It has been shown that in a multiple trapping model, the transit time of the carriers exhibits an electric field dependence

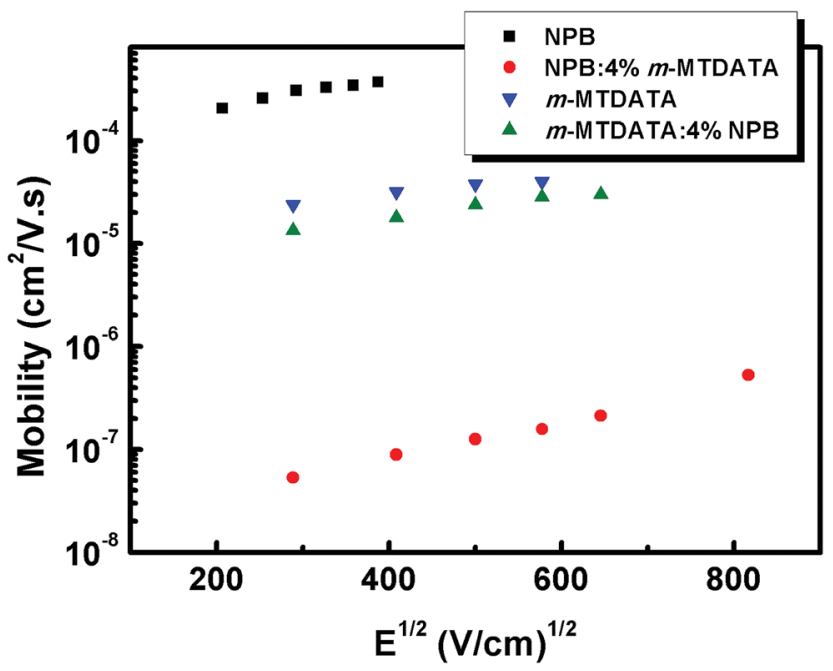

Fig. 3 Field dependent hole mobilities of NPB, NPB:m-MTDATA, $m$ MTDATA and $m$-MTDATA:NPB at room temperature.

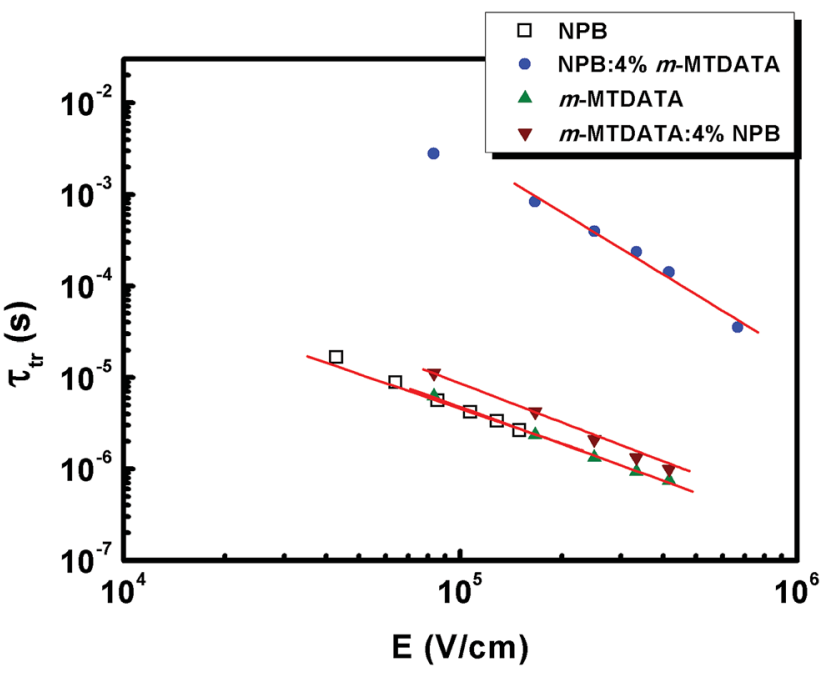

Fig. 4 Field dependence of hole transit times. The solid lines represent the linear fits to the data.

of $\tau_{\operatorname{tr}}(E) \propto E^{-1 / \alpha}$, where $\alpha$ is a dispersion parameter $(0<\alpha<1$, $\alpha=1$ for non-dispersive transport) that describes dispersive transport in amorphous solids. ${ }^{29}$ Generally, a more dispersive charge transport would result in a smaller value of $\alpha$. In addition, $\alpha$ is related to the expression $\alpha=T / T_{\mathrm{t}}$, where $T_{\mathrm{t}}$ is the characteristic temperature of trap distribution. The electric field dependence of the hole transit times derived from AS measurements are shown in Fig. 4. From the slope of the line representing the linear fit to the experimental data, the values of $\alpha$ and $T_{\mathrm{t}}$ obtained for different films are summarized in Table 1. It can be clearly seen that the $\alpha$ value of the NPB: $4 \% m$-MTDATA based device is the smallest, which suggests that the distribution of trap states in NPB:4\% $m$-MTDATA is the broadest. This result gives direct evidence of the trapping center that $\mathrm{m}$ MTDATA acts as when doped into NPB.

Moreover, the dispersive hole transport can be certified by a $C / C_{\text {geo }}-f / f_{\text {r }}$ plot as shown in Fig. 5 . For the NPB: $4 \% m$-MTDATA film, the curves for different bias voltages all have the same shape which again reflects the dispersive properties of hole transport. ${ }^{30}$ However, for the other films, the curves at different bias voltages have different shapes, indicating non-dispersive transport, which is in agreement with the results shown in Table 1.

To further distinguish the effect of trapping and scattering on the hole transport properties, the temperature dependent $I-$ $V$ characteristics of different doping devices are shown in Fig. 6. The slopes of the $I-V$ curves on a double logarithmic scale for

Table 1 The trap distribution parameters in pristine and doped films

\begin{tabular}{lllll}
\hline & $\alpha$ & $T_{\mathrm{t}}(\mathrm{K})$ & $V_{\mathrm{c}}(\mathrm{V})$ & $N_{\mathrm{t}}\left(\mathrm{cm}^{-3}\right)$ \\
\hline NPB & 0.79 & 379 & 11 & $2.5 \times 10^{17}$ \\
NPB:4\% m-MTDATA & 0.44 & 681 & 103 & $2.4 \times 10^{18}$ \\
$m$-MTDATA & 0.75 & 400 & 50 & $1.2 \times 10^{18}$ \\
$m$-MTDATA:4\% NPB & 0.71 & 422 & 78 & $1.8 \times 10^{18}$
\end{tabular}



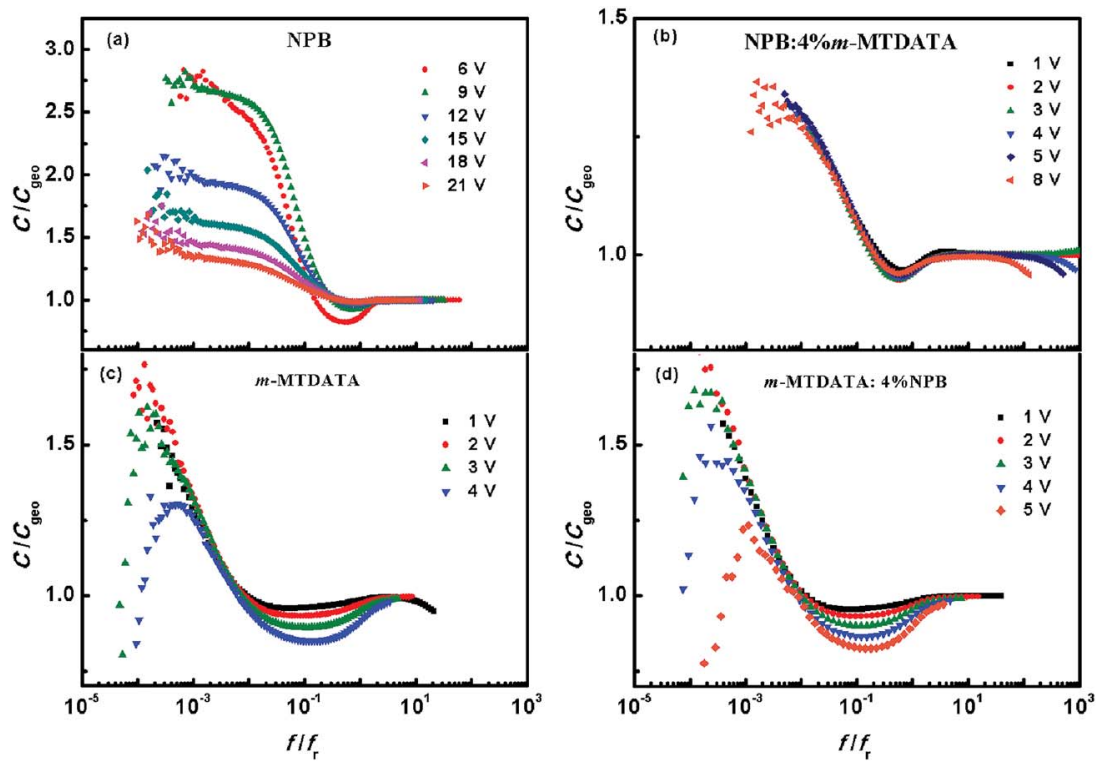

Fig. 5 Normalized capacitance $C / C_{\text {geo }}-f / f_{\mathrm{r}}$ curves for (a) NPB, (b) NPB:4\% m-MTDATA, (c) m-MTDATA, (d) $m$-MTDATA:4\% NPB.

each device are greater than 2 at room temperature and increase rapidly with decreasing temperature. Hence, the equation for $I-$ $V$ characteristics can be expressed $\mathrm{as}^{\mathbf{2 6}}$

$$
J=q^{1-l} \mu N_{\mathrm{v}}\left(\frac{2 l+1}{l+1}\right)^{l+1}\left(\frac{l}{l+1} \frac{\varepsilon \varepsilon_{o}}{N_{\mathrm{t}}}\right)^{l} \frac{V^{l+1}}{d^{2 l+1}}
$$

where $q$ is the elementary charge, $\mu$ is the hole mobility, $N_{\mathrm{v}}$ is the density of states in the valence band, $l$ is an energy parameter given by $T_{\mathrm{c}} / T$ ( $T_{\mathrm{c}}$ is the characteristic temperature of the traps), and $N_{\mathrm{t}}$ is the total density of the hole traps. As an analytical method introduced by Kumar et al. ${ }^{31}$ to determine $N_{\mathrm{t}}$, eqn (4) can be rearranged as follows:

$$
J=\frac{q \mu N_{\mathrm{v}} V}{d} f(l) \exp \left[-\frac{T_{\mathrm{c}}}{T} \ln \left(\frac{q d^{2} N_{\mathrm{t}}}{2 \varepsilon \varepsilon_{\mathrm{o}} V}\right)\right]
$$

with

$$
f(l)=\left(\frac{2 l+1}{l+1}\right)^{l+1}\left(\frac{l}{l+1}\right)^{l} \frac{1}{2^{l}}
$$
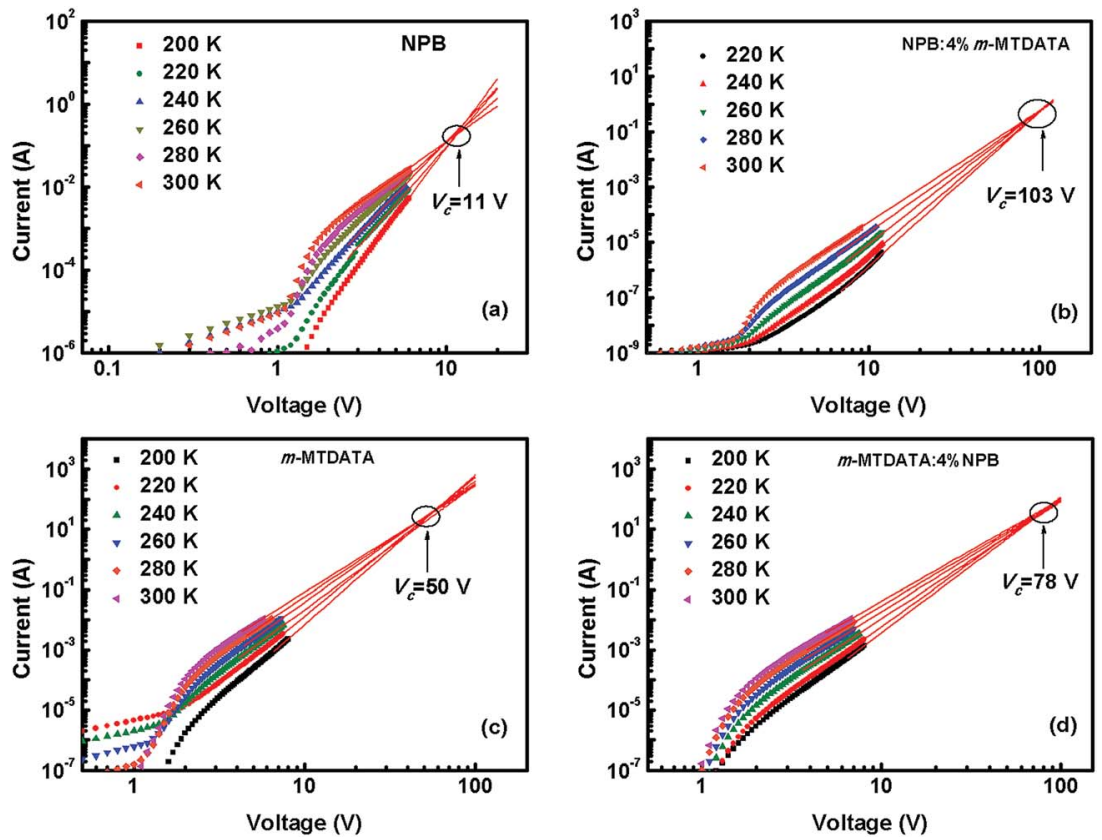

Fig. 6 Extrapolation of the $I-V$ curves at different temperatures in log-log scale for (a) NPB, (b) NPB:4\% $m$-MTDATA, (c) $m$-MTDATA, (d) $m$ MTDATA:4\% NPB hole-only devices. 

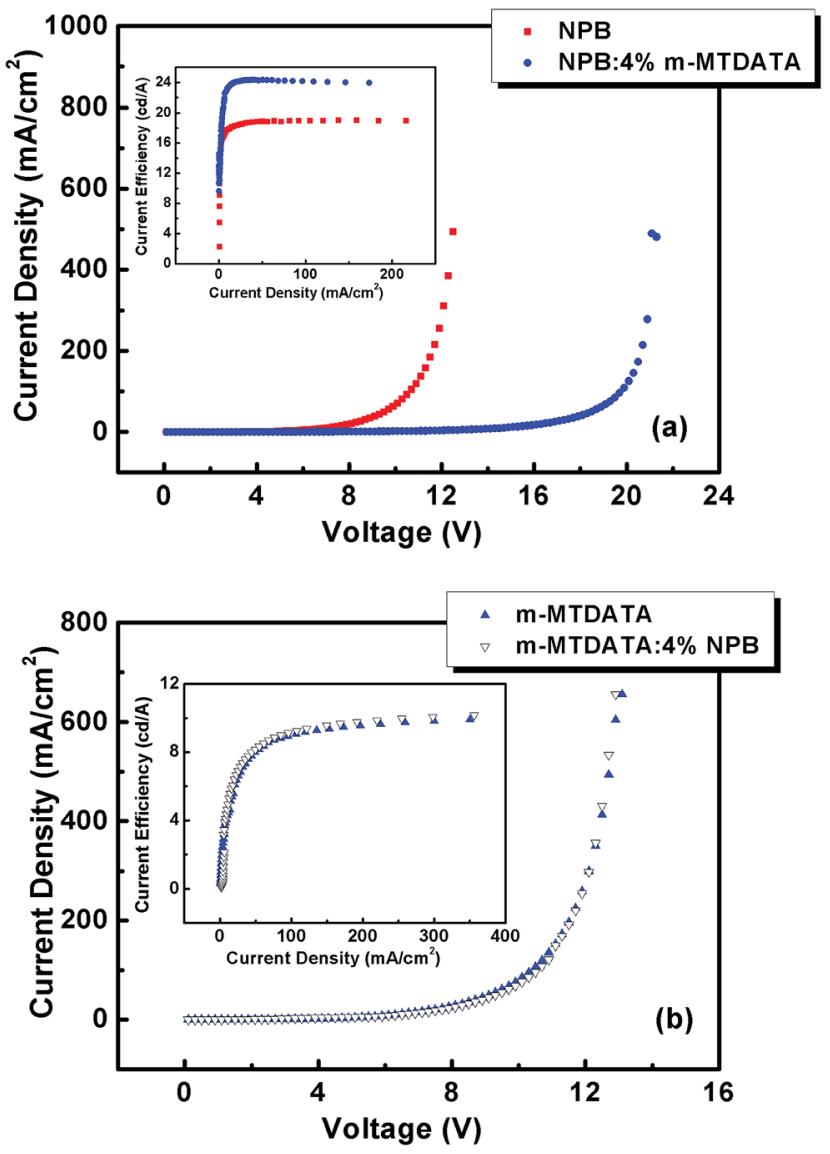

Fig. 7 Current density-voltage and current efficiency versus current density characteristics of OLEDs with (a) NPB, NPB: $4 \% m$-MTDATA and (b) $m$-MTDATA, $m$-MTDATA:4\% NPB as the hole transport layer.

For $l>2$, the value of $f(l)$ is about 0.5 , so it can be considered as a constant of 0.5 . With this approximation, eqn (5) becomes a typical Arrhenius form,

$$
J=J_{\mathrm{o}} \exp \left(-\frac{E_{\mathrm{a}}}{k T}\right)
$$

where

$$
E_{\mathrm{a}}=\frac{T_{\mathrm{c}}}{T} \ln \left(\frac{q N_{\mathrm{t}} d^{2}}{2 \varepsilon \varepsilon_{\mathrm{o}} V}\right)
$$

For $E_{\mathrm{a}}=0, J=J_{\mathrm{o}}$ and current becomes independent of temperature. The $I-V$ curves for different temperatures should then converge at a critical voltage,

$$
V_{\mathrm{c}}=\frac{q N_{\mathrm{t}} d^{2}}{2 \varepsilon \varepsilon_{\mathrm{o}}}
$$

By extrapolating $\log I-\log V$ characteristics at various temperatures to higher voltages, $V_{\mathrm{c}}$ can be determined, as shown in Fig. 6. With the obtained $V_{\mathrm{c}}$ and assuming $\varepsilon=3$, the total density of the traps can be calculated as listed in Table 1.

It appears that doping $m$-MTDATA into NPB raises the total density of traps by one order of magnitude, rendering the distribution of trap states broader. On the contrary, the scatterer NPB has a minimal affect on $m$-MTDATA with respect to the total density of traps. This result confirms the trapping activity of $m$-MTDATA in NPB.

Organic films were used as the hole-transporting layers (HTL) to fabricate organic light-emitting diodes with a general configuration of ITO/ $\mathrm{MoO}_{3}(5 \mathrm{~nm}) / \mathrm{HTL}(60 \mathrm{~nm}) / \mathrm{NPB}(20 \mathrm{~nm}) /$ $\mathrm{Alq}_{3}$ : 10-(2-benzothiazolyl)-2,3,6,7-tetrahydro-1,1,7,7-tetramethyl-1H,5H,11H-benzo[l]-pyrano-[6,7,8-ij]quinolizin-11-one $(\mathrm{C} 545 \mathrm{~T})(1 \%, 30 \mathrm{~nm}) / \mathrm{Alq}_{3}(30 \mathrm{~nm}) / \mathrm{LiF}(1 \mathrm{~nm}) / \mathrm{Al}$ or $\mathrm{ITO} / \mathrm{MoO}_{3}(5$ $\mathrm{nm}) / \mathrm{HTL}(60 \mathrm{~nm}) / m$-MTDATA $(20 \mathrm{~nm}) / \mathrm{Alq}_{3}$ : C545T (1\%, $\left.30 \mathrm{~nm}\right) /$ $\mathrm{Alq}_{3}(30 \mathrm{~nm}) / \mathrm{LiF}(1 \mathrm{~nm}) / \mathrm{Al}$ for doped NPB and $m$-MTDATA films, respectively. Inserting a layer of pure NPB or $m$-MTDATA between the HTL and the emitting layer will block electrons from reaching the HTL and make the interfaces between different HTLs and emitting layers the same. It can be observed from the data shown in Fig. 7 that the current density-voltage $(J-V)$ characteristics for OLEDs with trap doped HTLs differ strongly from those with scatterer doped HTLs. With balanced charge transport in $m$-MTDATA-doped NPB and $\mathrm{Alq}_{3}$, a maximum efficiency of $24.3 \mathrm{~cd} \mathrm{~A}^{-1}$ is achieved. ${ }^{32}$

\section{Conclusions}

In conclusion, the hole transport properties of blends of NPB and $m$-MTDATA compared with the pristine materials were investigated well using admittance spectroscopy and considering temperature dependent current-voltage characteristics and electroluminescent characteristics. The hole transport in NPB:4\% $m$-MTDATA shows more dispersion than that of the other films. The significant differences in trap density, trap distribution and carrier dispersion are responsible for a lowering of the hole mobility in trapping, which is quite distinct from the effect of scattering. These results pave the way for the facile tailoring of the charge transport properties by doping, thus shedding light on improving device performance.

\section{Conflicts of interest}

There are no conflicts to declare.

\section{Acknowledgements}

The authors gratefully acknowledge the Scientific Research Fund of Hunan Provincial Education Department (17C0331), the Hunan First Normal University Research Fund (XYS15N01) and the Natural Science Foundation of Hunan Province (Grant No. 2017JJ3046) for their support of this research. The authors also thank Lu She in the Hunan Provincial Tax Service, State Administration of Taxation for the encouragement.

\section{References}

1 C. W. Tang and S. A. Vanslyke, Appl. Phys. Lett., 1987, 51, 913915.

2 C. W. Tang, Appl. Phys. Lett., 1986, 48, 183-185. 
3 A. Tsumura, H. Koezuka and T. Ando, Appl. Phys. Lett., 1986, 49, 1210-1212.

4 Y. Zhang and H. Aziz, Org. Electron., 2016, 30, 76-82.

5 M. Hoping, C. Schildknecht, H. Gargouri, T. Riedl, M. Tilgner, H. H. Johannes and W. Kowalsky, Appl. Phys. Lett., 2008, 92, 213306.

6 Z. Chiguvare and V. Dyakonov, Phys. Rev. B: Condens. Matter Mater. Phys., 2004, 70, 235207.

7 D. Poplavskyy and F. So, J. Appl. Phys., 2006, 99, 033707.

8 Y. Yao, H. Dong and W. Hu, Adv. Mater., 2016, 28, 4513-4523. 9 Y. Wang, J. Chen, J. Huang, D. Ma, L. Dong and H. Chen, J. Appl. Phys., 2014, 115, 223707.

10 Y. Wang, J. Chen, L. Dong and D. Ma, J. Appl. Phys., 2013, 114, 113703.

11 Y. Wang, Q. Liang, J. Huang, D. Ma and Y. Jiao, RSC Adv., 2017, 7, 28494-28498.

12 R. Schmechel and H. von Seggern, Phys. Status Solidi A, 2004, 201, 1215-1235.

13 N. von Malm, J. Steiger, R. Schmechel and H. von Seggern, J. Appl. Phys., 2001, 89, 5559-5563.

14 S.-W. Liu, J.-H. Lee, C.-C. Lee, C.-T. Chen and J.-K. Wang, Appl. Phys. Lett., 2007, 91, 142106.

15 K. K. Tsung and S. K. So, Appl. Phys. Lett., 2008, 92, 103315. 16 K. L. Tong, S. W. Tsang, K. K. Tsung, S. C. Tse and S. K. So, J. Appl. Phys., 2007, 102, 093705.

17 C. Li, L. Duan, Y. Sun, H. Li and Y. Qiu, J. Phys. Chem. C, 2012, 116, 19748-19754.
18 P. M. Borsenberger and D. S. Weiss, Organic Photoreceptors for Imaging Systems, Dekker, New York, 1993.

19 K. K. Tsung and S. K. So, J. Appl. Phys., 2009, 106, 083710.

20 S. W. Tsang, S. K. So and J. B. Xu, J. Appl. Phys., 2006, 99, 013706.

21 S. W. Tsang, S. C. Tse, K. L. Tong and S. K. So, Org. Electron., 2006, 7, 474-479.

22 H. C. F. Martens, H. B. Brom and P. W. M. Blom, Phys. Rev. B: Condens. Matter Mater. Phys., 1999, 60, R8489-R8492.

23 H. C. F. Martens, J. N. Huiberts and P. W. M. Blom, Appl. Phys. Lett., 2000, 77, 1852-1854.

24 N. Shintaku, S. Izawa, K. Takagi, H. Naito and M. Hiramoto, Org. Electron., 2017, 50, 515-520.

25 L. Zhang, H. Nakanotani and C. Adachi, Appl. Phys. Lett., 2013, 103, 093301.

26 M. A. Lampert and P. Mark, Current Injection in Solids, Academic New York, 1970.

27 M. R. Khan, P. Anjaneyulu, K. S. R. Koteswara Rao and R. Menon, J. Phys. D: Appl. Phys., 2017, 50, 095103.

28 P. N. Murgatroyd, J. Phys. D: Appl. Phys., 1970, 3, 151-156.

29 H. Scher and E. W. Montroll, Phys. Rev. B: Solid State, 1975, 12, 2455-2477.

30 S. Berleb and W. Brütting, Phys. Rev. Lett., 2002, 89, 286601. 31 V. Kumar, S. C. Jain, A. K. Kapoor, J. Poortmans and R. Mertens, J. Appl. Phys., 2003, 94, 1283-1285.

32 S. Barth, P. Müller, H. Riel, P. F. Seidler, W. Rieß, H. Vestweber and H. Bässler, J. Appl. Phys., 2001, 89, 37113719 . 